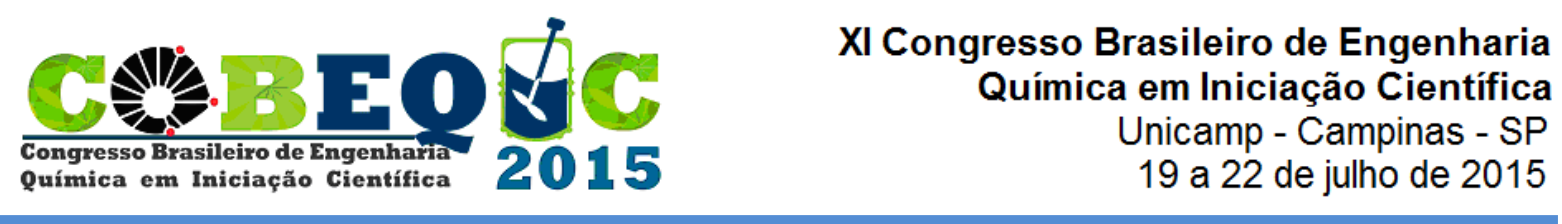

\title{
ESTUDO DA TRANSIÇÃO ENTRE ESCOAMENTO LAMINAR E TURBULENTO EM TUBO CAPILAR
}

\author{
M. H. MARTINS ${ }^{1}$, A. KNESEBECK ${ }^{1}$ \\ ${ }^{1}$ Universidade Federal do Paraná, Departamento de Engenharia Química \\ E-mail para contato: marcellohmartins@gmail.com
}

RESUMO - O número de Reynolds é definido pela relação entre as forças viscosas e inerciais de um fluido, e portanto, indica qual a importância dessas forças para seu escoamento. Mas uma pequena variação das forças que definem Reynolds pode causar grande alteração nesse coeficiente, mudando a forma de escoamento e, portanto, suas características. A zona de transição entre escoamento laminar e turbulento, porém, é complexa e não se tem completo entendimento de seus fenômenos. Esse trabalho objetiva determinar experimentalmente o valor de transição entre os regimes laminar e turbulento em um tubo capilar escoando ar, bem como a aplicação dos modelos para queda de pressão nesse sistema. $\mathrm{O}$ valor de Reynolds encontrado para a transição foi de 2360 , dentro da faixa esperada. O modelo laminar foi adequado para representar os pontos experimentais, enquanto que o modelo do turbulento não refletiu a tendência na região da transição.

\section{INTRODUÇÃO}

O escoamento laminar é aquele no qual o fluido se move em camadas ou lâminas e há grande influência das forças viscosas. No escoamento turbulento, as partículas fluidas se misturam ao longo do deslocamento, devido à flutuações aleatórias no campo de velocidades. Nesse caso as forças viscosas são desprezíveis em relação às forças de inércia.

O escoamento turbulento pode ou não ser desejável. No caso do deslocamento de dois fluidos em que necessita a ocorrência de mistura, esse tipo de deslocamento é favorável. No deslocamento de sangue nas artérias, o deslocamento turbulento contribui para que todas as partículas do sangue cheguem às paredes arteriais e realizem as trocas necessárias (Fox et al., 2014). Porém em outros casos a turbulência é indesejada, porque cria maior resistência ao escoamento, além da análise desse tipo de escoamento ser mais complexa devido a presença de flutuações aleatórias de velocidade e de alta frequência. Um número adimensional que relaciona as forças viscosas e inerciais na condução do fluido é o número de Reynolds $(R e)$, Equação 1. Esse número é utilizado em inúmeras aplicações em que o transporte de quantidade de movimento está envolvido.

$$
\operatorname{Re}=\frac{\rho v D}{\mu}
$$

$\mathrm{Na}$ Equação 1, o numerador representa as forças inerciais e o denominador as forças viscosas. Assim, o aumento da viscosidade $\mu$ ou a diminuição do diâmetro $D$ do tubo, quando a velocidade é a mesma, resulta em um decaimento do valor de Reynolds, e valores menores do coeficiente indicam um escoamento laminar, enquanto os valores maiores geralmente estão 


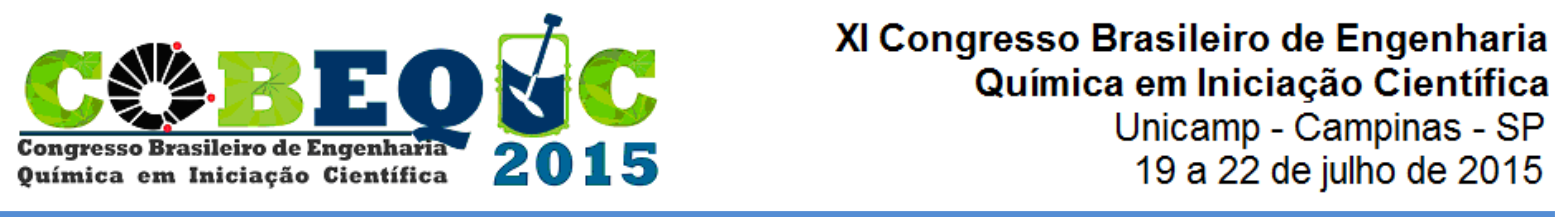

ligados a um escoamento turbulento. O número normalmente assumido como valor de transição, para fins de cálculo, é $R e=2300$.

Nesse estudo foram realizadas determinações experimentais da condução de ar em um capilar, relacionando a velocidade $v$ de escoamento com a queda de pressão $\Delta P$. Esse estudo permite a determinação do valor de Reynolds em que a transição ocorre, caracterizada como o ponto onde a relação entre a queda de pressão e a velocidade entre a queda de pressão e a velocidade deixa de ser linear. É feita a comparação entre a queda de pressão experimental e a prevista por modelos teóricos, no intuito de averiguar a aplicação desses modelos para o sistema em questão. A queda de pressão devido ao escoamento de um fluido em uma tubulação pode ser modelada a partir da Equação 2 (Fox et al., 2014).

$$
\Delta P=f \frac{L}{D} \frac{v^{2}}{2}
$$

Nessa equação, $f$ é o fator de atrito do escoamento e $L$ é o comprimento do tubo. No caso de regime laminar, a partir das equações de Navier-Stokes, demonstra-se que a queda de pressão pode ser obtida pela Equação 3, (Bird et al., 2007):

$$
\Delta P=\frac{32 \mu L v}{D^{2}}
$$

Nessa equação, observa-se que existe uma relação linear entre a queda de pressão e a velocidade de escoamento do fluido. Também se pode observar que a queda de pressão é diretamente proporcional à viscosidade do fluido, mas não é função de sua densidade $(\rho)$, o que caracteriza a predominância das forças viscosas em relação às inerciais.

Comparando-se as Equações 2 e 3 e considerando a definição do número de Reynolds (Equação 1), chega-se à forma do fator de atrito para escoamento laminar, Equação 4:

$$
f=\frac{64}{\operatorname{Re}}
$$

Quando se inicia a turbulência no escoamento, a relação entre a queda de pressão e a velocidade do escoamento deixa de ser linear e a Equação 4 não se aplica. Nesse caso, é necessária a determinação experimental do fator de atrito, que é dependente da rugosidade da tubulação e pode ser determinada experimentalmente. Se a rugosidade puder ser desprezada, o tubo é considerado liso, e nesse caso uma das equações mais utilizadas é a equação de Blasius, (Fox et al, 2014):

$$
f=\frac{0,316}{\operatorname{Re}^{0,25}} \quad \operatorname{Re}<10^{5}
$$

\section{MATERIAIS E MÉTODOS}


Um esquema da instalação utilizada está mostrado na Figura 1. A vazão do ar vindo do compressor é regulada por meio da válvula agulha, passando para um integralizador calibrado de volume para o cálculo da velocidade do fluido. Em seguida o ar é enviado ao capilar, sendo que na entrada deste a pressão $P p$ é mensurada por um sensor de pressão tipo tubo em "U". Para os menores valores de queda de pressão, utiliza-se um tubo "U" inclinado, aumentando a precisão dos dados coletados.

Figura 1 - Módulo experimental.

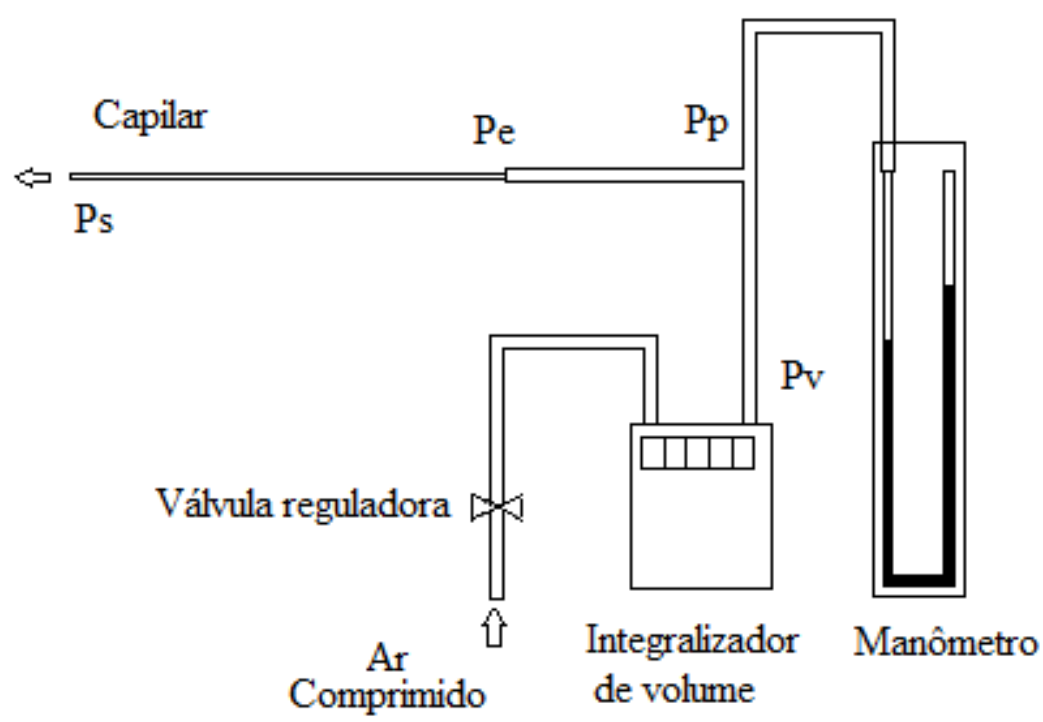

O fluido utilizado é ar comprimido. As determinações foram conduzidas nas condições ambientes a uma temperatura de $15^{\circ} \mathrm{C}$, e a pressão de $685 \mathrm{mmHg}$, sendo que a densidade e viscosidade do ar foram consideradas como $1,11 \mathrm{~kg} / \mathrm{m}^{3}$ e $1,79.10^{-5} \mathrm{~Pa}$.s respectivamente. Considerando-se gás ideal, a partir da Equação 6 pode-se calcular a densidade do ar nos pontos $P v$, para a determinação da vazão mássica, e $P e$, para a determinação da queda de pressão total no capilar, a partir da pressão e uma densidade de referência.

$$
\frac{\rho 1}{\rho 2}=\frac{P 1}{P 2}
$$

O fluido utilizado no manômetro, para medidas da variação de pressão $(\Delta \mathrm{P})$ foi água a $15^{\circ} \mathrm{C}$, usando $\rho=998 \mathrm{~kg} / \mathrm{m}^{3}$. O tubo capilar, de vidro, tem diâmetro de $2,83 \mathrm{~mm}$, obtido por volumetria com água, e seu comprimento é $1,21 \mathrm{~m}$. Por ser feito de vidro é considerado tubo liso.

O manômetro mede a pressão relativa na entrada do capilar. Como o "tê" de vidro onde está inserido o sensor de pressão tem diâmetro maior que o do capilar, é necessário calcular a pressão que se desenvolve na entrada do capilar devido à variação da energia cinética. Para isso, foi aplicada a equação de Bernoulli, resultando na Equação 7, na qual os índices $V$ e $E$ referem-se, respectivamente, à saída do integralizador e entrada do capilar. 


$$
P_{E}=P_{V}-\frac{\rho v_{V}^{2}}{2}\left[\left(\frac{D_{V}}{D_{E}}\right)^{4}-1\right]
$$

Para a obtenção da queda de pressão distribuída no capilar é necessário descontar as quedas de pressão localizadas $\Delta P_{L}$ devido à entrada e à saída do tubo. Para isso utilizou-se a equação contida no trabalho de Morini et al. (2006), Equação 8. A queda de pressão distribuída $\Delta \mathrm{P}$ foi encontrada subtraindo a localizada da total, Equação 9.

$$
\begin{aligned}
& \Delta P_{L}=\left(k_{e}+k_{s}\right) \frac{\rho v^{2}}{2} \\
& \Delta P=\Delta P_{E}-\Delta P_{L}
\end{aligned}
$$

Na Equação 8, $k_{e}$ e $k_{s}$ são chamados respectivamente de menor coeficiente de perda na entrada e menor coeficiente de perda na saída. Seus valores são respectivamente 1,5 e 1 (Morini et al., 2006). A Equação 8 pode ser usada dessa forma porque o gás, nas condições do experimento, é considerado incompressível, uma vez que a sua velocidade de escoamento é menor que 0,3 vezes a velocidade do som. A velocidade do ar na saída do integralizador de volume é diferente da velocidade do ar no tubo capilar, devido à mudança de pressões e do diâmetro da tubulação. Essa velocidade pode ser calculada a partir de dados do ar na saída do integralizador e da pressão na entrada do capilar, a partir da equação de Bernoulli, resultando na Equação 10. Com essa velocidade, o número de Reynolds é calculado pela Equação 1 com os parâmetros da entrada do capilar.

$$
v_{E}=v_{v} \frac{P_{V}}{P_{E}}\left(\frac{D_{V}}{D_{E}}\right)^{2}
$$

\section{RESULTADOS E DISCUSSÕES}

\subsection{Perdas de carga localizada e distribuída}

A queda de pressão experimental total foi diminuída da queda de pressão localizada usando as Equações 8 e 9, resultando na queda de pressão distribuída. A Figura 2 mostra os valores encontrados para as quedas de pressão total e distribuída.

Figura 2 - Valores experimentais de queda de pressão total e distribuída 


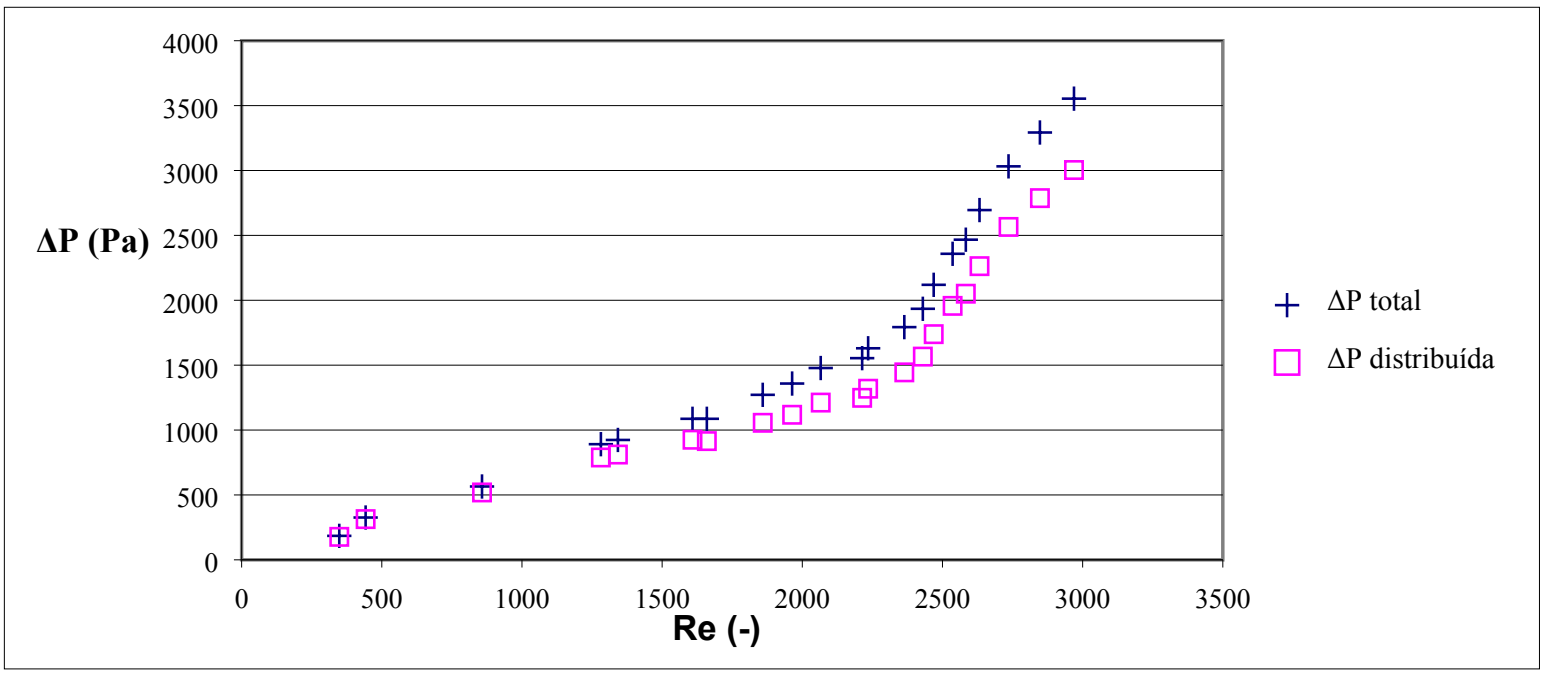

Na Figura 2 observa-se que, conforme a velocidade do gás aumenta, as curvas entre a queda de pressão total e a distribuída se distanciam. Para baixos Re, a queda de pressão localizada é desprezível, porém esta aumenta significativamente à medida que Re aumenta. Isso reflete o fato que, usando a Equação 8, a queda de pressão é dependente do quadrado da velocidade do gás, ou seja, sua influência é maior à medida que a velocidade aumenta.

\subsection{Aplicação dos modelos de queda de pressão}

A Figura 3 compara os dados experimentais de queda de pressão distribuída com a prevista pelos modelos de regime laminar, Equação 4, e regime turbulento, Equação 5. A partir dessa figura nota-se nitidamente o momento em que o regime deixa de ser laminar, caracterizado por uma relação linear de queda de pressão, e passa a crescer de forma a acompanhar os valores do regime turbulento. $\mathrm{O}$ valor do número de Reynolds onde ocorre a mudança de regime é 2360. Esse valor é compatível com os valores de literatura, de cerca de 2100 (Bird et al., 2007) a 2300 (Fox et al., 2014). Nesse ponto, o $\Delta \mathrm{P}$ do capilar é $1277 \mathrm{~Pa}$.

Figura 3 - Comparação dos valores das quedas de pressão experimentais e teóricas 


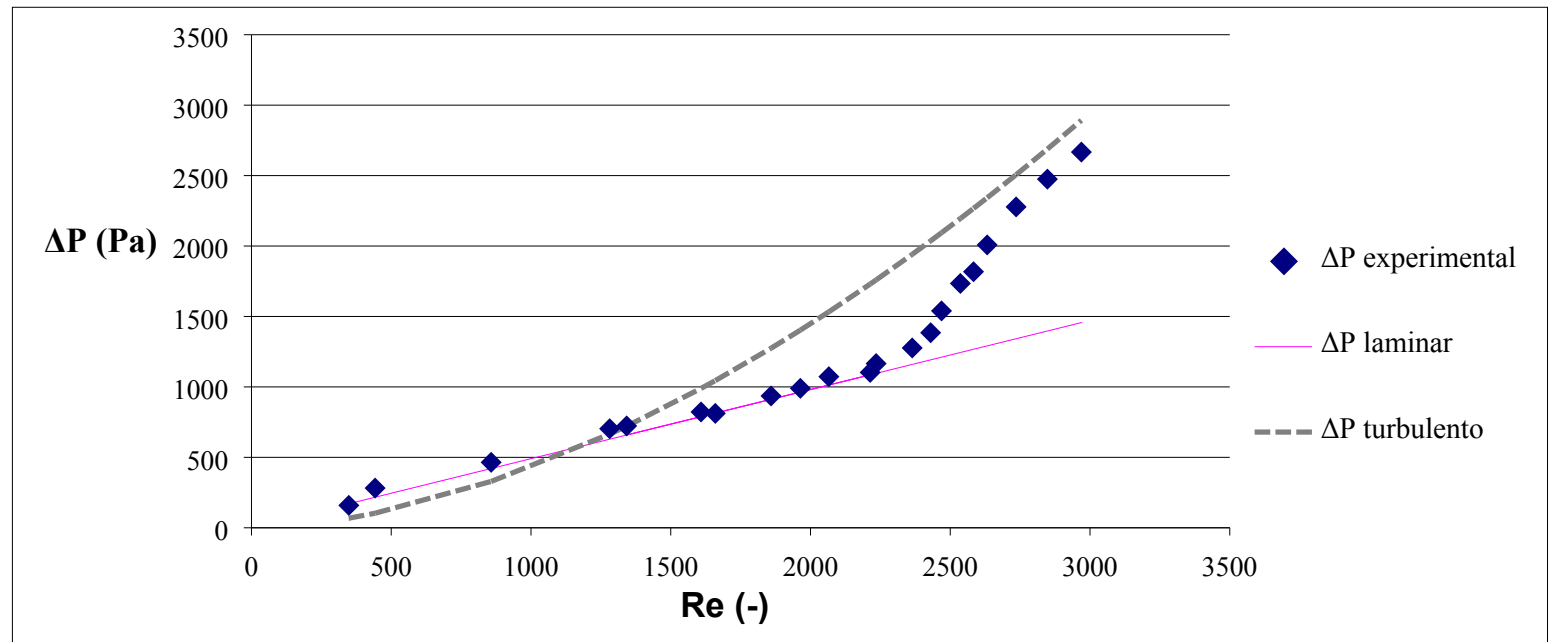

O modelo para o regime laminar, Equação 4, tem resultados excelentes quando comparado com os dados experimentais. Para o regime turbulento, calculado pela Equação 5, o modelo aproxima-se dos pontos experimentais à medida que o número de Reynolds aumenta. Na região de Reynolds entre 2360 e 2740, porém, o modelo turbulento não consegue acompanhar a tendência dos valores de queda de pressão, o que sugere a necessidade do desenvolvimento de equações específicas para essa região de transição.

\section{CONCLUSÕES}

O valor de Reynolds encontrado para a transição entre o regime laminar e turbulento foi de 2360 para uma variação de pressão de $1277 \mathrm{~Pa}$. Esse valor é obtido como o ponto em que a curva de queda de pressão em função do número de Reynols deixa de ser linear. $\mathrm{O}$ modelo para o regime laminar apresentou excelentes resultados comparado com os valores experimentais. Isso indica que as pressões calculadas na entrada do capilar, quando se considera as conversões de energia através da equação de Bernoulli, são adequadas, bem como o cálculo das variações de pressão na entrada e na saída do tubo. O modelo para a queda de pressão no regime turbulento aproximou-se dos dados experimentais para as maiores vazões utilizadas, porém na região da transição ele não reflete bem a tendência dos pontos experimentais.

\section{REFERÊNCIAS}

BIRD, R. B.; STEWART, W. E.; LIGHTFOOT, E. N. Transport Phenomena, New York, John Willey, 2007.

FOX, R.W.; MCDONALD, A.T.; PRITCHARD, P.J. Introdução à mecânica dos fluidos. Rio de Janeiro: Editora LTC, 2014.

MORINI, G.L.; LORENZINI, M.; SALVIGNI, S. Friction characteristics of compressible gas flows in microtubes. Experimental thermal and fluid science v. 30, p. 733$744,2006$. 